\title{
Role of serine/threonine kinase 33 methylation in colorectal cancer and its clinical significance
}

\author{
MING-DI YIN, SI-PING MA, FANG LIU and YU-ZE CHEN \\ Department of Colon and Rectum Surgery, Cancer Hospital of China Medical University, \\ Liaoning Cancer Hospital and Institute, Shenyang, Liaoning 110042, P.R. China
}

Received July 6, 2016; Accepted November 7, 2017

DOI: $10.3892 / \mathrm{ol} .2017 .7614$

\begin{abstract}
Serine/threonine kinase 33 (STK33) is a novel protein that has been the focus of an increasing number of studies in recent years; however, the role of STK33 in tumorigenesis remains controversial. Previous studies have demonstrated that STK33 is overexpressed in several human cancers and exerts a pro-tumorigenic effect through the promotion of cell proliferation. However, the role of STK33 in colorectal cancer (CRC), which is one of the most aggressive human malignancies, remains unclear. The aim of the current study was to investigate the methylation status of STK33 in CRC and to determine its clinical significance. The results demonstrated that STK33 was hypermethylated in $\mathrm{CRC}$ cell lines and promoted the proliferation of CRC cells. In addition, the methylation status and expression of STK33 in 94 pairs of cancer and noncancerous tissues obtained from patients with CRC was investigated. STK33 methylation was significantly increased in cancer tissues when compared with adjacent noncancerous tissues $(\mathrm{P}<0.001)$. STK33 methylation was associated with lymph node metastasis $(\mathrm{P}<0.05)$, tumor invasion $(\mathrm{P}<0.05)$, distant metastases $(\mathrm{P}<0.01)$ and tumor stage $(\mathrm{P}<0.01)$. Reduced STK33 mRNA and protein expression in CRC was associated with STK33 hypermethylation $(\mathrm{P}<0.001)$. In addition, patients with hypermethylated STK33 exhibited shorter overall survival rates when compared with those with unmethylated STK33 $(\mathrm{P}<0.01)$. In conclusion, the results of the current study suggest that STK33 hypermethylation may be a promising novel biomarker for the diagnosis, prognosis and suitable treatment of CRC.
\end{abstract}

Correspondence to: Dr Si-Ping Ma, Department of Colon and Rectum Surgery, Cancer Hospital of China Medical University, Liaoning Cancer Hospital and Institute, 44 Xiaoheyan Road, Dadong, Shenyang, Liaoning 110042, P.R. China

E-mail: siping_ma@yeah.net

Key words: serine/threonine kinase 33, methylation, colorectal cancer, prognostic, calcium/calmodulin-dependent kinase

\section{Introduction}

Globally, colorectal cancer (CRC) is the third most commonly diagnosed cancer in males and the third most commonly diagnosed cancer in females according to a previous epidemiological survey data (1). It is also one of the leading causes of cancer-associated mortality worldwide (1). Each year, $\sim 1$ million new cases are diagnosed and $30 \%$ of the 600,000 people who succumb to the disease each year reside in China $(2,3)$. Over the past ten years, the incidence and mortality rates of CRC have rapidly increased due to the prevalence of obesity, which could partially have attributed to the development of China's economy (4). Despite developments in diagnostic and therapeutic strategies, which have already improved the survival rates of patients with early stage CRC, the prognosis of patients with late stage CRC remains poor $(5,6)$. Therefore, further investigations are required to gain an improved understanding of the molecular characteristics and associated biological mechanisms underlying the proliferation, migration and metastasis of CRC cells. This may also enable the identification of early screening markers and therapeutic targets.

The human serine/threonine kinase 33 (STK33) enzyme belongs to calcium/calmodulin-dependent kinase family and is located on chromosome 11p15.3, which is a gene-rich region associated with several diseases, including cancer (7). A previous study investigating the expression of STK33 mRNA and protein in normal human adult and embryonic tissues demonstrated that STK33 is expressed in a variety of normal tissues but at very low levels (8). However, it was observed to be highly expressed in the testis, particularly in the spermatogenic epithelium. It has also been demonstrated that STK33 involved in the 'synthetic lethality' process in a variety of tumor cells, which occurs when deficiency in the expression of multiple genes results in cell death and depends on the Ras oncogene (9). This finding implies that STK33 may serve a significant role in molecular targeted therapy for KRAS-dependent tumors (9). By contrast, a different study demonstrated that the activity of STK33 may be nonessential in KRAS-dependent cell lines (10). Therefore, the role of STK33 in tumor cells remains controversial and the mechanisms underlying the function of STK33 in tumor biology are complex. Previous studies have demonstrated that STK33 is overexpressed in hypopharyngeal squamous cell carcinoma (HSCC) (11), hepatocellular carcinoma (HCC) (12) and human 
large cell lung cancer (LC) (13), and the increased expression of STK33 may subsequently promote tumorigenesis and disease progression. However, previous research aiming to identify hypermethylated genes in CRC, demonstrated that the STK33 gene is hypermethylated and its expression is subsequently downregulated when compared with normal colorectal tissues (14). This is inconsistent with the results of the aforementioned studies and therefore requires further investigation.

To date, the role of STK33 in CRC tumorigenesis and its clinical significance remains unclear. Therefore, the aim of the current study was to investigate the methylation status of STK33 in CRC cell lines and patients with CRC. The expression of STK33 mRNA and protein in the collected tumor tissues and its association with cell proliferation was also investigated. In addition, the association between STK33 methylation status and the clinicopathological characteristics of patients with CRC was evaluated to determine the clinical significance of STK33 in CRC.

\section{Materials and methods}

Patients and samples. A total of 94 patients (46-72 years old, 49 males and 45 females) with CRC who underwent surgical treatment at the Liaoning Cancer Hospital \& Institute (Shenyang, China) between October 2007 and May 2010, were recruited to this study. In order to be included in the study, patients were required to have not received any prior anticancer therapies, including chemotherapy, radiotherapy or surgery prior to enrollment to the present study. Follow up began the day after the surgery and lasted for 60 months. Fresh tumor tissues and adjacent non-cancerous tissues obtained from patients with CRC were frozen in liquid nitrogen following resection and stored at $-80^{\circ} \mathrm{C}$ for subsequent experiments. The clinicopathological features of each patient are shown in Table I. Tumor grades were defined in accordance with the criteria of the World Health Organization (15). The pathological tumor, node, metastasis (TNM) status of all CRC cases was defined according to the criteria of the sixth edition of the TNM classification of the International Union Against Cancer (2002) (16). The current study was approved by the Ethics Committee of Liaoning Cancer Hospital \& Institute, and written informed consent was obtained from all recruited patients.

Cell lines. The normal colon cell line, NCM460, and DLD-1 and HCT-116 CRC cell lines were obtained from the American Type Culture Collection (Manassas, VA, USA). The NCM460 cell line was cultured in complete Dulbecco's modified Eagle's medium (Gibco; Thermo Fisher Scientific, Inc., Waltham, MA, USA), and the CRC cell lines were cultured in RPMI-1640 medium (Gibco; Thermo Fisher Scientific, Inc.). All media was supplemented with $10 \%$ fetal bovine serum (Gibco; Thermo Fisher Scientific, Inc.) and $1 \%$ streptomycin and penicillin (Sigma-Aldrich; Merck KGaA, Darmstadt, Germany). All cells were cultured in a humidified $37^{\circ} \mathrm{C}$ incubator supplemented with $5 \% \mathrm{CO}_{2}$.

Genomic DNA extraction. Genomic DNA from the NCM460, DLD-1 and HCT-116 cell lines and fresh frozen tissues was extracted using the QIAamp DNA Mini kit (Qiagen, Inc., Valencia, CA, USA) according to the manufacturer's protocols, and the procedures described previously (14). Briefly, cell pellets harvested from cell culture medium through centrifugation for $2 \mathrm{~min}$ at $6,000 \mathrm{xg}$ and $4^{\circ} \mathrm{C}$, and tissue samples minced using 3-mm diameter grinders, were mixed with $700 \mu 1$ lysis buffer containing $20 \mu \mathrm{g} / \mathrm{ml}$ protease K (Sigma-Aldrich; Merck $\mathrm{KGaA}$ ), $20 \mathrm{mM}$ Tris $\mathrm{HCl}$ (pH 8.0), 5 mM EDTA (pH 8.0), $400 \mathrm{mM} \mathrm{NaCl}$, and 1\% SDS solution (Sigma-Aldrich; Merck $\mathrm{KGaA}$ ). The mixture was then incubated at $42^{\circ} \mathrm{C}$ for $\sim 12 \mathrm{~h}$. Genomic DNA was purified using the phenol/chloroform extraction method. Purified DNA was then eluted in $100 \mu 1$ water and quantified using a Thermo Scientific NanoDrop 2000/2000C Spectrophotometer (Thermo Fisher Scientific, Inc., Pittsburgh, PA, USA).

Quantitative methylation-specific polymerase chain reaction (QMSP). The genomic DNA obtained from the cell lines and fresh frozen tissues was bisulfite-treated using the EpiTect fast DNA bisulfite kit (Qiagen, Inc.), in order to convert any unmethylated cytosine residues in $\mathrm{CpG}$ islands into uracil residues, while any non-methylated cytosines remained unchanged. All procedures were performed according to the manufacturer's protocols. The reaction mixture consisted of $85 \mu \mathrm{l}$ bisulfite mix solution and $35 \mu \mathrm{l}$ DNA protection buffer, and was incubated at room temperature for $30 \mathrm{~min}$. The bisulfite-converted genomic DNA was eluted from columns

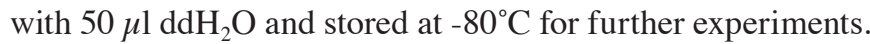

The methylation status of the bisulfite-converted genomic DNA was measured by methylation-specific PCR using the Applied Biosystems 7500 Real-Time PCR System (Thermo Fisher Scientific, Inc.) according to the manufacturer's protocol. The primer pairs used to detect methylation status were as follows: STK33, forward, 5'-GTGCGTATTTGTCGG AGATTC-3', and reverse, 5'-TACCATAACAACGACCTA ACCG-3'; $\beta$-actin, forward, 5'-TGGTGATGGAGGAGGTTT AGTAAGT-3', and reverse, 5'AACCAATAAAACCTACTC CTCCCTTAA-3'. Reactions were performed using PCR vials containing $15 \mu 12 X$ Maxima SYBR Green/ROX qPCR Master Mix (Thermo Fisher Scientific, Inc.), $250 \mathrm{nM}$ each forward and reverse primer, $30 \mathrm{ng}$ bisulfite-converted genomic DNA template and $\mathrm{ddH}_{2} \mathrm{O}$ to a final volume of $30 \mu \mathrm{l}$. Experiments were performed in triplicate for each sample. The QMSP program was set as follows: Denaturation at $94^{\circ} \mathrm{C}$ for $3 \mathrm{~min}$ followed by 40 cycles at $94^{\circ} \mathrm{C}$ for $15 \mathrm{sec}, 60^{\circ} \mathrm{C}$ for $10 \mathrm{sec}$ and $72^{\circ} \mathrm{C}$ for $10 \mathrm{sec}$. There was an additional extension step at $72^{\circ} \mathrm{C}$ for 7 min prior to the completion of PCR. Relative quantification of the amplified gene levels in the bisulfite-converted DNA sample was achieved by measuring the quantification cycle $\left(\mathrm{C}_{\mathrm{q}}\right)$ values of STK33 and $\beta$-actin and applying the $2^{-\Delta \Delta C q}$ method (17). PCR products were loaded directly onto $3 \%$ agarose gels and visualized with ethidium bromide. The gel was visualized using a gel image analysis system (JC-300; Shanghai Peiqing Science and Technology Co., Ltd., Shanghai, China).

STK33 RNA inhibition. The expression of STK33 was knocked down in NCM460, DLD-1 and HCT-116 cell lines using an STK33-RNA inference (STK33-RNAi) vector constructed by Shanghai GeneChem Co, Ltd. (Shanghai, China). The STK33 
Table I. Association between STK33 methylation and the clinicopathological features of patients with colorectal cancer.

\begin{tabular}{|c|c|c|c|c|}
\hline \multirow[b]{2}{*}{ Variable } & \multirow[b]{2}{*}{ No. of cases } & \multicolumn{2}{|c|}{ STK33 methy lation status } & \multirow[b]{2}{*}{ P-value } \\
\hline & & Methylated & Unmethylated & \\
\hline \multicolumn{5}{|l|}{ Gender } \\
\hline Male & 49 & 30 & 19 & NS \\
\hline Female & 45 & 27 & 18 & \\
\hline \multicolumn{5}{|c|}{ Age (years) } \\
\hline$\geq 50$ & 54 & 29 & 25 & NS \\
\hline$<50$ & 40 & 26 & 14 & \\
\hline \multicolumn{5}{|c|}{ Tumor location } \\
\hline Colon & 59 & 38 & 21 & NS \\
\hline Rectum & 35 & 17 & 18 & \\
\hline \multicolumn{5}{|c|}{ Lymph node metastases } \\
\hline Absent & 58 & 39 & 19 & 0.041 \\
\hline Present & 36 & 16 & 20 & \\
\hline \multicolumn{5}{|c|}{ Tumor invasion } \\
\hline $\mathrm{T} 1 / \mathrm{T} 2$ & 52 & 30 & 22 & 0.032 \\
\hline $\mathrm{T} 3 / \mathrm{T} 4$ & 42 & 25 & 17 & \\
\hline \multicolumn{5}{|c|}{ CEA level } \\
\hline No & 50 & 31 & 19 & NS \\
\hline Yes & 44 & 24 & 20 & \\
\hline \multicolumn{5}{|c|}{ Distant metastasis } \\
\hline Absent & 63 & 43 & 20 & 0.006 \\
\hline Present & 31 & 12 & 19 & \\
\hline \multicolumn{5}{|c|}{ Tumor stage } \\
\hline $\mathrm{I}-\mathrm{II}$ & 65 & 30 & 35 & 0.002 \\
\hline III-IV & 29 & 25 & 4 & \\
\hline
\end{tabular}

STK33, serine/threonine kinase 33; NS, not significant; CEA, carcinoembryonic antigen.

small interfering RNA (siRNA) oligonucleotide sequences were as follows: Forward, 5'-GATCCCAGAGAATGAGAC AAGGTGTTCAAGAGACACCTTGTCTCATTCTCTGAGA-3', and reverse, 5'-AGCTTCTCAGAGAATGAGACAAGGTGT CTCTTGAACACCTTGTCTCATTCTCTGG-3'. The plasmid was transfected into cells according to a previously published protocol (12). Following transfection with Lipofectamine 2000 (Invitrogen; Thermo Fisher Scientific, Inc., Waltham, MA, USA) for $24 \mathrm{~h}$, cells were washed with PBS for reverse transcription-quantitative polymerase chain reaction (RT-qPCR) analysis and the cell proliferation assay. The cells without siRNA transfection were used as controls.

$R T-q P C R$. Total RNA from cell lines and fresh frozen tissues was isolated using TRIzol reagent (Thermo Fisher Scientific, Inc.) according to the manufacturer's protocols. Total RNA was eluted in $20 \mu \mathrm{l}$ DEPC-treated water and the concentration was determined using the Thermo Fisher NanoDrop 2000/2000C Spectrophotometer (Thermo Fisher Scientific, Inc.). cDNA was synthesized from $1 \mu \mathrm{g}$ RNA obtained from each sample using the PrimeScript RT Reagent kit (Takara Biotechnology Co., Ltd., Dalian, China). The reaction mixture consisted of $1 \mu \mathrm{g}$ RNA, $4 \mu 1$ 5X PrimeScript buffer, $1 \mu 150 \mu \mathrm{M}$ oligo-dT

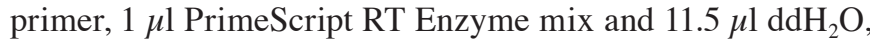
and the reaction was conducted at $37^{\circ} \mathrm{C}$ for $1 \mathrm{~h}$. cDNA was eluted with $\mathrm{ddH}_{2} \mathrm{O}$ and stored at $-80^{\circ} \mathrm{C}$.

qPCR was performed to determine the expression of STK33 in cell lines and fresh frozen tissues using SYBR Green qPCR SuperMix UDG with ROX kit (Invitrogen; Thermo Fisher Scientific, Inc.). The Applied Biosystems 7500 Real-Time PCR System (Thermo Fisher Scientific, Inc.) was used and the following thermal cycling parameters were applied: $95^{\circ} \mathrm{C}$ for $10 \mathrm{~min}$ followed by 35 cycles of $95^{\circ} \mathrm{C}$ for $10 \mathrm{sec}$ and $60^{\circ} \mathrm{C}$ for $1 \mathrm{~min}$. The primers used were as follows: STK33, forward, 5'-CTTCGGTGAGACCAACCAAT-3' and reverse, 5'-TGT AATTGGCATCAGGGACA-3'; and $\beta$-actin, forward, 5'-AGA GCTACGAGCTGCCTGAC-3' and reverse, 5'-AGCACTGTG TTGGCGTACAG-3'. The expression of $\beta$-actin was used as an endogenous standard to normalize the expression of STK33 using the $2^{-\Delta \Delta \mathrm{Cq}}$ method (17). Each sample was tested at least 3 times simultaneously.

Western blot analysis. Total proteins were extracted from cell lines and fresh tissues using radioimmunoprecipitation assay lysis buffer (Santa Cruz Biotechnology, Inc., Dallas, TX, USA) and centrifuged at $14,000 \times \mathrm{g}$ for $10 \mathrm{~min}$ at $4^{\circ} \mathrm{C}$ 
A

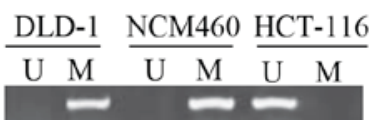

$\mathrm{C}$

DLD-1 NCM460 HCT-116
B

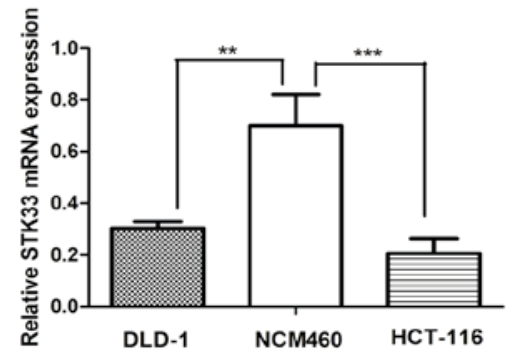

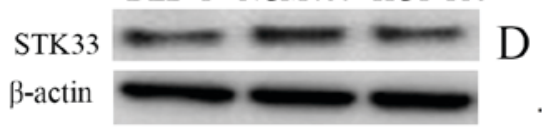
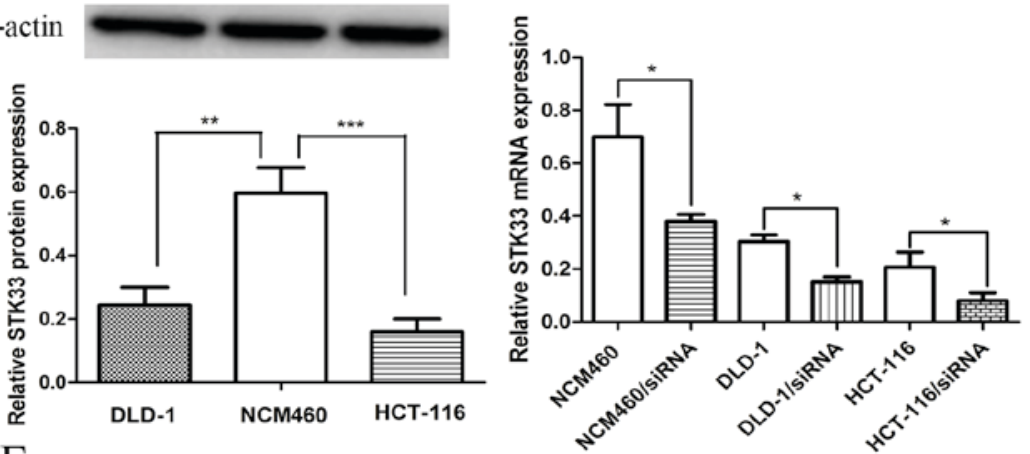

$\mathrm{E}$

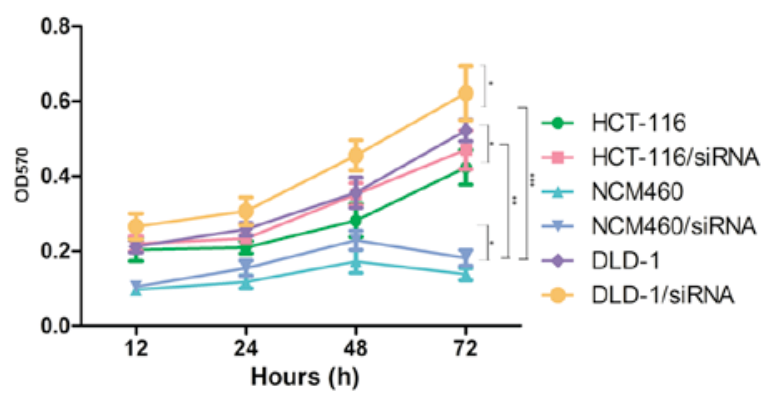

Figure 1. Characterization of STK33 gene methylation status and STK33 expression in the DLD-1 and HCT-116 colorectal cancer cell lines and normal NCM460 cells. (A) Ethidium bromide staining of amplified bisulfite-treated DNA samples analyzed by quantitative methylation-specific polymerase chain reaction. Lanes M, MSP for methylated promoter, lanes U, MSP for unmethylated promoter. (B) Reverse transcription-quantitative polymerase chain reaction was used to determine the expression of STK33 mRNA. (C) Western blot analysis was used to determine the expression of STK33 protein. (D) Reverse transcription-quantitative polymerase chain reaction analysis was used to determine the expression of STK33 in STK33-small interfering RNA transfected $\mathrm{CRC}$ and normal colon cell lines. (E) An MTT assay was used to analyze the rate of cell proliferation. Values are presented as the mean \pm standard deviation. Experiments were performed in triplicate. ${ }^{* * *} \mathrm{P}<0.001,{ }^{* *} \mathrm{P}<0.01$ and ${ }^{*} \mathrm{P}<0.05$ as indicated. STK33, serine/threonine kinase 33 ; OD, optical density.

to obtain crude protein extracts. The concentration of the protein extracts was measured by BCA Protein Assay Kit (Thermo Fisher Scientific Inc.). Protein samples $(50 \mu \mathrm{g})$ were separated by $10 \%$ sodium dodecyl sulfate-polyacrylamide gel electrophoresis and then transferred onto a PVDF membrane. The membrane was blocked with 5\% skimmed milk at room temperature for $1 \mathrm{~h}$ and then incubated with the following primary antibodies: STK33 mouse anti-human monoclonal antibody (dilution, 1:1,000; cat no. ab57693) and $\beta$-actin mouse anti-human monoclonal antibody (dilution, 1:1,000; cat no. ab8226; both Abcam, Cambridge, MA, USA) at $4^{\circ} \mathrm{C}$ for $\sim 12 \mathrm{~h}$. The membrane was then incubated with horseradish peroxidase-conjugated goat anti-mouse secondary antibody (1:1,000; cat no. ab97040; Abcam) at room temperature for $2 \mathrm{~h}$. The bands were visualized using enhanced chemiluminescence reagent (EMD Millipore, Billerica, MA, USA) and quantified by densitometry analysis using ImageJ software (version 1.48; National Institutes of Health, Bethesda, MD, USA).
Cell proliferation assay. An MTT assay was performed to measure the proliferation of the normal colon and CRC cell lines, as well as STK33-RNAi transfected cells in the current study. Briefly, cells were seeded in a 96 -well plate at a cell density of $3 \times 10^{3}$ cells $/ \mathrm{ml}$ for $24 \mathrm{~h}$. A total of $20 \mu \mathrm{l} \mathrm{MTT} \mathrm{solu-}$ tion ( $5 \mathrm{mg} / \mathrm{ml}$; Sigma-Aldrich; Merck KGaA) was then added to each well at indicated time points $(12,24,48$ and $72 \mathrm{~h})$, and cells were incubated for $4 \mathrm{~h}$ at $37^{\circ} \mathrm{C}$. Following incubation, the cell culture medium was removed and $150 \mu \mathrm{l}$ DMSO was added to each well to dissolve the formazan crystals. The optical density was measured at a wavelength of $570 \mathrm{~nm}$ using a Thermo Multiskan Spectrum spectrophotometer (Thermo Fisher Scientific, Inc.). Each measurement was performed in triplicate under the same conditions.

Immunohistochemistry analysis (IHC). IHC analysis was performed on the frozen tumor and adjacent normal tissues obtained from patients with CRC. Tissues were fixed in $4 \%$ formalin at room temperature for $48 \mathrm{~h}$ and embedded in 

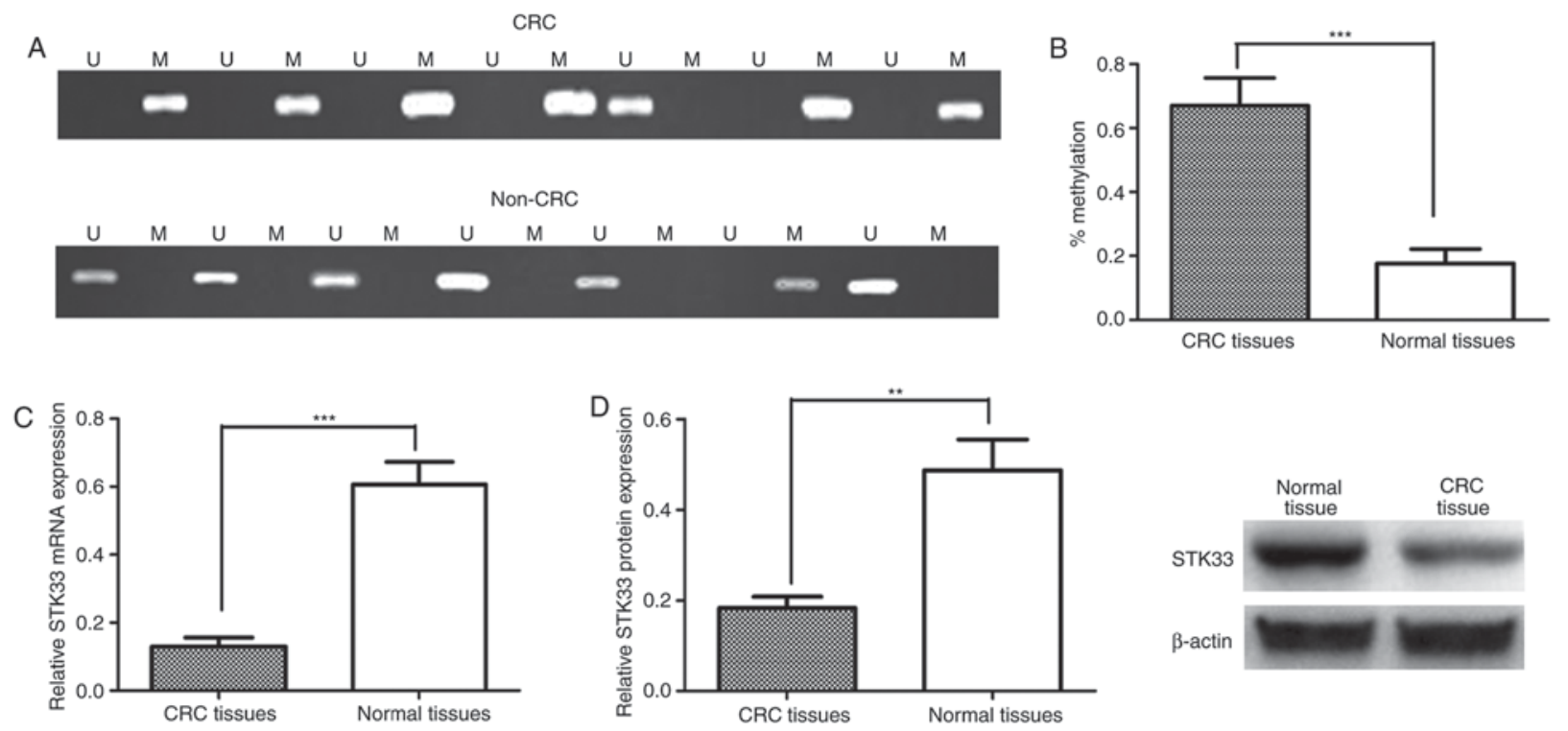

Figure 2. Characterization of STK33 gene methylation status and STK33 expression in CRC tissues and adjacent normal tissues from patients with CRC. (A) Ethidium bromide staining of amplified bisulfite-treated DNA samples analyzed by quantitative methylation-specific polymerase chain reaction. (B) Quantification of STK33 gene methylation levels. (C) Reverse transcription-quantitative polymerase chain reaction analysis was used to determine the expression of STK33. (D) Western blot analysis was used to determine the expression of STK33 protein. Values are presented as the mean \pm standard deviation. Experiments were performed in triplicate. ${ }^{* * *} \mathrm{P}<0.001$ and ${ }^{* *} \mathrm{P}<0.01$ as indicated. STK33, serine/threonine kinase 33; CRC, colorectal cancer; M, methylated promoter; $\mathrm{U}$, unmethylated promoter.

paraffin. The paraffin-embedded tissues $(5 \mu \mathrm{m})$ were then deparaffinized in $100 \%$ xylene (Sigma-Aldrich; Merck $\mathrm{KGaA}$ ) and rehydrated using a graded ethanol series. Tissues were subsequently washed using citrate buffer (pH 6.0) 3 times (1 min each time) and incubated with 5\% goat serum (Beyotime, Shanghai, China) for $30 \mathrm{~min}$ at $37^{\circ} \mathrm{C}$. Following washing with PBS, sections were incubated with the primary mouse anti-human STK33 monoclonal antibody for $24 \mathrm{~h}$ at $4^{\circ} \mathrm{C}$ (dilution, 1:1,000; cat no. ab57693), followed by the horseradish peroxidase conjugated goat anti-mouse secondary antibody (dilution, 1:1,000; cat no. ab97040; both Abcam) for $2 \mathrm{~h}$ at $4^{\circ} \mathrm{C}$. All sections were counterstained with $0.2 \%$ hematoxylin for $5 \mathrm{~min}$ at room temperature, dehydrated with ethanol and washed with $100 \%$ xylene for $10 \mathrm{sec}$ at room temperature. The Olympus CX31-LV320 microscope (Olympus Corporation, Tokyo, Japan) was used to observe the stained tissues.

Statistical analysis. The results were analyzed using GraphPad Prism 6.0 statistical software (GraphPad Software, Inc. La Jolla, CA, USA). Data are presented as the mean \pm standard deviation. A Student's t-test or one-way analysis of variance followed by Tukey's test were conducted to determine the significance between or among different groups. A $\chi^{2}$ test and Fisher's exact test were used to examine the association of STK33 methylation and various clinicopathological parameters. Overall survival was analyzed using the Kaplan-Meier method and the statistical significance between survival curves was assessed by log-rank test. Univariate Cox proportional hazard regressions were applied to estimate the individual hazard ratios for overall survival. The variables that were significant in the univariate analysis were included in the multivariate analysis. $\mathrm{P}<0.05$ was determined to indicate a statistically significant difference.

\section{Results}

STK33 is hypermethylated in CRC cell lines. The methylation status of STK33 in CRC cell lines was investigated by performing QMSP in normal colon cells, NCM460, and CRC cell lines, DLD-1 and HCT-116. The STK33 in CRC cell lines was methylated while STK33 in normal colon cell was unmethylated (Fig. 1A). The expression of STK33 mRNA and protein in NCM460, DLD-1 and HCT-116 cells was then determined to investigate the effect of STK33 hypermethylation on STK33 expression. The expression of STK33 mRNA and protein was significantly decreased in the DLD-1 (both $\mathrm{P}<0.01$ ) and HCT-116 $(\mathrm{P}<0.001)$ cell lines compared with NCM460 cells (Fig. 1B and C). These results suggest that STK33 may serve a crucial role in the development of CRC, and may also present a useful biomarker.

STK33 hypermethylation may promote cell proliferation. The hypermethylation status of STK33 in CRC cell lines DLD-1 and HCT-116 was established through QMSP analysis. The results indicated that STK33 hypermethylation may lead to the downregulation of STK33 at the mRNA and protein expression levels. siRNA was used to knock down the expression of STK33 in NCM460, DLD-1 and HCT-116 cell lines. The results of RT-qPCR demonstrated that STK33 siRNA efficiently downregulated the expression of STK33 in these cell lines (Fig. 1D). The role of STK33 hypermethylation on the progression and development of CRC was investigated by analyzing the cell proliferation rate of CRC and normal colon cells, as well as the STK33-downregulated cell lines, using an MTT assay. The results demonstrated that the cell proliferation rate of DLD-1 and HCT-116 CRC cells was increased compared with normal NCM460 cells (Fig. 1E). The cell proliferation rate of cells with RNAi-induced knockdown of 
Table II. Univariate analysis of the association between STK33 methylation and mRNA expression levels.

\begin{tabular}{lccc}
\hline & \multicolumn{2}{c}{ STK33 expression } & \\
\cline { 2 - 3 } Methylation status & $\begin{array}{c}\text { Downregulated } \\
(\mathrm{n}=51)\end{array}$ & $\begin{array}{c}\text { No change } \\
(\mathrm{n}=43)\end{array}$ & P-value \\
\hline Methylated (n=55) & 38 & 17 & $<0.001$ \\
Unmethylated (n=39) & 13 & 26 & \\
\hline
\end{tabular}

STK33, serine/threonine kinase 33.

Table III. Univariate and multivariate analysis of the association between clinical features and overall survival.

A, Univariate analysis

\begin{tabular}{lccc}
\hline Variable & HR & $95 \%$ CI & P-value \\
\hline STK33 & 2.055 & $1.145-3.687$ & 0.016 \\
Age & 1.555 & $0.867-2.787$ & 0.138 \\
Gender & 1.490 & $0.826-2.688$ & 0.185 \\
Tumor location & 1.174 & $0.967-3.039$ & 0.065 \\
Lymph node metastases & 2.059 & $1.147-3.695$ & 0.015 \\
Tumor invasion & 1.878 & $1.059-3.331$ & 0.031 \\
CEA level & 1.631 & $0.915-2.908$ & 0.097 \\
Distant metastasis & 1.961 & $1.100-3.498$ & 0.022 \\
Tumor stage & 2.163 & $1.198-3.905$ & 0.011 \\
\hline
\end{tabular}

B, Multivariate analysis

\begin{tabular}{lccc}
\hline Variable & HR & $95 \%$ CI & P-value \\
\hline STK33 & 2.147 & $1.189-3.874$ & 0.011 \\
Age & - & - & - \\
Gender & - & - & - \\
Tumor location & - & - & - \\
Lymph node metastases & 1.963 & $1.101-3.502$ & 0.022 \\
Tumor invasion & 1.796 & $1.018-3.166$ & 0.043 \\
CEA level & - & - & - \\
Distant metastasis & 2.057 & $1.146-3.690$ & 0.016 \\
Tumor stage & 2.061 & $1.149-3.699$ & 0.015 \\
\hline
\end{tabular}

STK33, serine/threonine kinase 33; CEA, carcinoembryonic antigen; $\mathrm{HR}$, hazard ratio, CI, confidence interval.

STK33 expression was significantly increased when compared with the untransfected cells ( $\mathrm{P}<0.01$, Fig. 1E). Taken together these results suggest that the hypermethylation of STK33 in CRC cell lines may promote CRC cell proliferation.

STK33 hypermethylation in patients with CRC. The potential role of STK33 in the development and progression of CRC was investigated further by determining the methylation status of STK33 in 94 pairs of CRC tissues and adjacent noncancerous
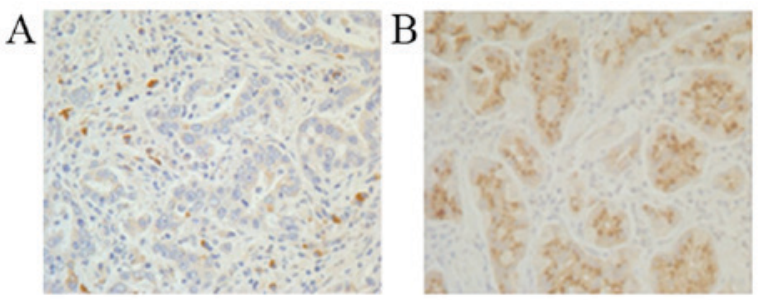

Figure 3. Immunohistochemical analysis of the expression of serine/threonine kinase 33 in (A) colorectal cancer tissues and (B) adjacent noncancerous tissues. x200, magnification.

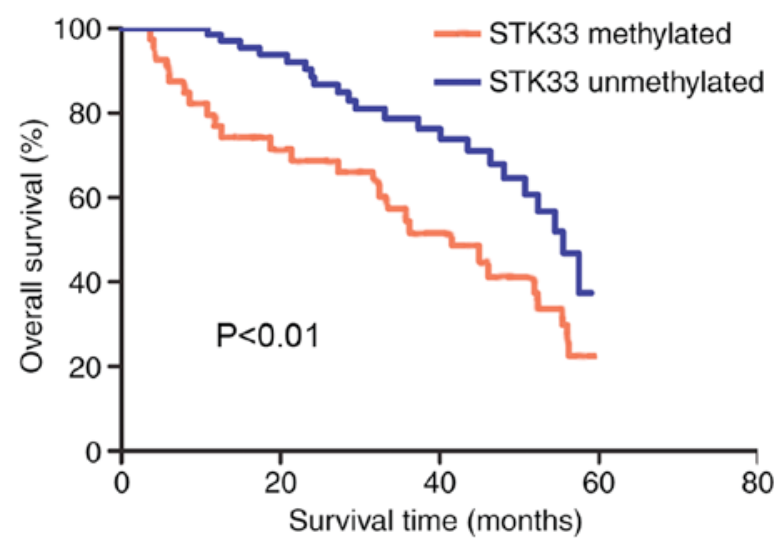

Figure 4. Kaplan-Meier survival curve of colorectal cancer patients with methylated and unmethylated STK33. STK33, serine/threonine kinase 33.

tissues obtained from patients with CRC enrolled in the current study. STK33 was observed to be hypermethylated in 55/94 (58.51\%) CRC tissue samples and 18/94 adjacent noncancerous tissue samples (19.15\%; Fig. 2A). The methylation status of STK33 in CRC and adjacent noncancerous tissue samples was then compared. The results demonstrated that methylation of STK33 was significantly increased in CRC tissues when compared with adjacent noncancerous tissues $(\mathrm{P}<0.001$; Fig. 2B). The expression of STK33 mRNA and protein in $\mathrm{CRC}$ and adjacent noncancerous tissues was then measured using RT-qPCR and western blot analysis, respectively, to examine the effect of hypermethylation on the expression of STK33. The expression of STK33 mRNA and protein was significantly decreased in CRC tissues compared with adjacent noncancerous tissues $(\mathrm{P}<0.001$ and $\mathrm{P}<0.01$, respectively; Fig. 2C and D). The association between STK33 hypermethylation and mRNA expression was also investigated (Table II). The results indicated that downregulation of STK 33 mRNA was associated with STK33 hypermethylation $(\mathrm{P}<0.001)$.

To investigate the expression and localization of STK33 in CRC tissues, IHC analysis of the CRC and adjacent noncancerous tissues was performed. Consistent with the conclusions drawn from the RT-qPCR and western blot analyses, STK33 was downregulated in CRC tissues compared with adjacent noncancerous tissues (Fig. 3). The results suggest that hypermethylation of STK33 in patients with CRC may be responsible for the reduced expression of STK33 at the mRNA and protein levels.

Clinical significance of STK33 hypermethylation in CRC. The clinical significance of STK33 hypermethylation in 
CRC was determined by investigating the association between STK33 hypermethylation and the clinicopathological parameters recorded for patients with CRC. The results indicated the STK33 hypermethylation in CRC samples was associated with lymph node metastasis $(\mathrm{P}<0.05)$, tumor invasion $(\mathrm{P}<0.05)$, distant metastasis $(\mathrm{P}<0.01)$ and $\mathrm{TNM}$ stage $(\mathrm{P}<0.01$; Table $\mathrm{I})$. There was no association observed between STK33 hypermethylation and the remaining clinicopathological features, including age, gender, tumor location and carcinoembryonic antigen (CEA) level (Table I).

STK33 hypermethylation is associated with poor prognosis in $C R C$. The overall survival rate of patients with hypermethylated and unmethylated STK33 was compared using the Kaplan-Meier method. The survival curves demonstrated that patients with hypermethylated STK33 exhibited a significantly shorter survival rate compared with those with unmethylated STK33 ( $\mathrm{P}<0.01$; Fig. 4). The univariate analysis demonstrated that STK33 hypermethylation, lymph node metastasis, tumor invasion, distant metastasis and TNM stage were all associated with the overall survival of patients with $\mathrm{CRC}$ (all $\mathrm{P}<0.05$; Table III). However, no association between overall survival and age, gender, tumor location and CEA level were observed. Multivariate Cox regression analysis was then used to analyze significant associations from the univariate analysis. The results demonstrated that STK33 hypermethylation, lymph node metastasis, tumor invasion, distant metastasis and TNM stage were independent predictive indicators of a poor outcome in CRC (all $\mathrm{P}<0.05$; Table III). The results therefore indicate that STK33 hypermethylation may present a useful prognostic marker for CRC.

\section{Discussion}

STK33. encodes a human kinase enzyme that was discovered by comparative genomic analysis of human chromosome $11 \mathrm{p} 15.3$ and its orthologous region on the distal region of mouse chromosome 7 (7). The STK33 gene consists of 12 exons and a 1,545 bp open reading frame, which was identified from the full-length transcript amplified from human uterus RNA (7). STK33 is differentially expressed in a variety of normal and malignant tissues (18). Protein kinases serve a major role in the regulation of a number of fundamental cellular processes (19). The location of STK33 in the human genome is strongly associated with many diseases (20); therefore, studies aiming to interpret the role of STK33 expression in cancer have been conducted (9). It has been demonstrated that STK33 is overexpressed in HSCC, HCC and LC (10-13). However, the STK33 gene has been demonstrated to be hypermethylated and in CRC cell lines, and its mRNA expression levels have been observed to be downregulated in tumor tissues compared with normal tissues (14). These results are not consistent with the results demonstrated in HSCC, HCC and LC. The role of STK33 in CRC is currently unknown; therefore, the aim of the present study was to investigate the methylation status of STK33 in patients with CRC, and examine its potential as a novel diagnostic and therapeutic target.

The present study demonstrated that the STK33 gene was hypermethylated in CRC cell lines, which is inconsistent with previous research (14). RT-qPCR and western blotting analyses demonstrated that the expression of STK33 mRNA and protein in CRC cell lines was significantly reduced compared with that in the normal colon cell line. The expression of STK33 in the cell lines was then knocked down using siRNA, which was subsequently verified by RT-qPCR. The effect of STK33 hypermethylation on CRC progression was investigated by measuring the cell proliferation rate of the CRC and normal colon cell lines. The cell proliferation rate was increased in the CRC cell lines when compared with normal colon cells, which suggests that STK33 hypermethylation may promote cell proliferation. However, the mechanisms underlying these effects require further study. It should be noted that the limitation of the in vitro experiment of the present study was the lack of scramble or negative control siRNA transfected groups. The cells transfected with siRNA targeting STK33 were used to investigate the effect of STK33 on cell proliferation and those without siRNA transfection were used as control.

The methylation of STK33 and its clinical significance in 94 patients with CRC was then investigated. The STK33 gene was hypermethylated in the majority of tissues from patients with $\mathrm{CRC}$, and further statistical analysis indicated that the expression of STK33 mRNA and protein was significantly decreased in CRC tissues with hypermethylated STK33 when compared with those with unmethylated STK33. The results also indicated that reduced STK33 expression was associated with STK33 hypermethylation. Therefore, gene methylation may be a major mechanism for the silencing of STK33 expression in CRC. IHC analysis also confirmed the results obtained from the RT-qPCR and western blotting analysis demonstrating that STK33 expression was downregulated in CRC tissue samples with STK33 hypermethylation.

The analysis of the association between STK33 hypermethylation and clinicopathological features demonstrated that lymph node metastasis, tumor invasion, distant metastasis and TNM stage were significantly associated with STK33 hypermethylation. During the patient follow-up period, it was observed that the overall survival of patients with hypermethylated STK33 was decreased compared with those with unmethylated STK33. This suggests that STK33 hypermethylation may be associated with a poor outcome for patients with CRC. Furthermore, the univariate and multivariate analyses indicated that STK33 hypermethylation, lymph node metastasis, tumor invasion, distant metastasis and TNM stage were independent predictive factors for the poor prognosis of CRC.

In conclusion, the results of the present study demonstrated that the STK33 gene was hypermethylated in CRC cell lines and tissue samples when compared with normal controls, which may be responsible for the observed reduction in STK33 mRNA and protein expression levels. In addition, the results suggest that STK33 hypermethylation may present a useful biomarker for the prognosis of patients with CRC. To the best of the author's knowledge, this is the first study to investigate the hypermethylation status of STK33 in CRC, and explore the association between STK33 hypermethylation and clinicopathological features. Limitations of the present study include the small number of enrolled patients. Therefore, future studies should include more patients to verify the 
findings we obtained. Furthermore, more research is required to further elucidate the mechanism of how STK33 promotes CRC progression.

\section{References}

1. Siegel R, Desantis C and Jemal A: Colorectal cancer statistics, 2014. CA Cancer J Clin 64: 104-117, 2014.

2. Li S, Wang J, Lu Y and Fan D: Screening and early diagnosis of colorectal cancer in China: A 12 year retrospect (1994-2006) J Cancer Res Clin Oncol 133: 679-686, 2007.

3. Zhao P, Dai M, Chen W and Li N: Cancer trends in China. Jpn J Clin Oncol 40: 281-285, 2010.

4. Chen WQ, Zheng R, Baade PD, Zhang S, Zeng H, Bray F, Jemal A, Yu XQ and He J: Cancer statistics in China, 2015. CA Cancer J Clin 66: 115-132, 2016.

5. Li L and Ma BB: Colorectal cancer in Chinese patients: Current and emerging treatment options. Onco Targets Ther 7: 1817-1828, 2014.

6. Jemal A, Bray F, Center MM, Ferlay J, Ward E and Forman D: Global cancer statistics. CA Cancer J Clin 61: 69-90, 2011.

7. Mujica AO, Hankeln T and Schmidt ER: A novel serine/threonine kinase gene, STK33, on human chromosome 11p15.3. Gene 280: 175-181, 2001.

8. Mujica AO, Brauksiepe B, Saaler-Reinhardt S, Reuss S and Schmidt ER: Differential expression pattern of the nove serine/threonine kinase, STK33, in mice and men. FEBS J 272: 4884-4898, 2005.

9. Scholl C, Fröhling S, Dunn IF, Schinzel AC, Barbie DA, Kim SY, Silver SJ, Tamayo P, Wadlow RC, Ramaswamy S, et al: Synthetic lethal interaction between oncogenic KRAS dependency and STK33 suppression in human cancer cells. Cell 137: 821-834, 2009.

10. Babij C, Zhang Y, Kurzeja RJ, Munzli A, Shehabeldin A, Fernando M, Quon K, Kassner PD, Ruefli-Brasse AA, Watson VJ, et al: STK33 kinase activity is nonessential in KRAS-dependent cance. Cancer Res 71: 5818-5826, 2011
11. Lingyan Huang LY, Chen C, Zhang GD, Ju YR, Zhang JZ, Wang HB and Li JF: STK33 overexpression in hypopharyngeal squamous cell carcinoma: Possible role in tumorigenesis. BMC Cancer 15: 13, 2015.

12. Wang P, Chen H, Wu J, Yan A and Zhang L: STK33 plays an important positive role in the development of human large cell lung cancers with variable metastatic potential. Acta Biochim Biophys Sin (Shanghai) 47: 214-223, 2015.

13. Yang T, Song B, Zhang J, Yang GS, Zhang H, Yu WF, Wu MC, Lu JH and Shen F: STK33 promotes hepatocellular carcinoma through binding to c-Myc. Gut 65: 124-133, 2016.

14. Moon JW, Lee SK, Lee JO, Kim N, Lee YW, Kim SJ, Kang HJ, Kim J, Kim HS and Park SH: Identification of novel hypermethylated genes and demethylating effect of vincristine in colorectal cancer. J Exp Clin Cancer Res 33: 4, 2014.

15. Rindi G, Arnold R, Bosman FT, Bosman T, Carneiro F, Hruban R and Theise N: Nomenclature and classification of neuroendocrine neoplasms of the digestive system WHO classification of tumours of the digestive system 4th edition. Int Agen Res Cancer (IARC): 13-14, 2010.

16. Greene FL, Page DL, Fleming ID, Fritz A, Balch CM and Haller DG: AJCC cancer staging handbook from the AJCC cancer staging manual. 6th edition. Springer, New York, 2002.

17. Livak KJ and Schmittgen TD: Analysis of relative gene expression data using real-time quantitative PCR and the 2(-Delta Delta C(T)) method. Methods 25: 402-408, 2001.

18. Bastienne B, Mujica AO, Herrmann H and Schmidt ER: The Serine/threonine kinase Stk33 exhibits autophosphorylation and phosphorylates the intermediate filament protein Vimentin. BMC Biochem 9: 25, 2008.

19. Wilmanns M, Gautel $M$ and Mayans O: Activation of calcium/calmodulin regulated kinases. Cell Mol Biol (Noisy-le-grand) 46: 883-894, 2000.

20. Nowak NJ and Shows TB: Genetics of chromosome 11: Loci for pediatric and adult malignancies, developmental disorders, and other diseases. Cancer Invest 13: 646-659, 1995.

This work is licensed under a Creative Commons Attribution-NonCommercial-NoDerivatives 4.0 International (CC BY-NC-ND 4.0) License. 